\title{
Atomic-resolution Observations of Grain Boundary Segregation in Multiphase Ceramics by Aberration-corrected STEM
}

\author{
Komal Syed, Mingjie Xu, David Kok, Kenta Ohtaki, William Bowman and Martha Mecartney \\ University of California-Irvine, Irvine, California, United States
}

Bulk ceramic processing includes the densification of powders at high temperature. This study evaluates elemental segregation behavior at grain boundaries for a three-phase ceramic $\mathrm{Al}_{2} \mathrm{O}_{3}-\mathrm{MgAl}_{2} \mathrm{O}_{4}-8$ mole $\% \quad \mathrm{Y}_{2} \mathrm{O}_{3}$ stabilized cubic $\mathrm{ZrO}_{2}$ (YSZ) made under different processing conditions: (1) conventional sintering, (2) flash sintering, and (3) spark plasma sintering (SPS) ${ }^{[1,2]}$. Understanding the elemental segregation at grain boundaries and point defect generation in the bulk can provide practical knowledge of processing for desirable properties using grain boundary engineering.

TEM samples were prepared by using a TESCAN GAIA3 FIB-SEM. State-of-the-art aberration-corrected JEOL Grand ARM300CF TEM/STEM, operated at $300 \mathrm{keV}$, was used to characterize the grain boundaries. The electron beam probe size was $\sim 0.1 \mathrm{~nm}$ with a probe current of about $35 \mathrm{pA}$. Low-background, high count analytical TEM holder was used to minimize any stray $\mathrm{x}$-rays caused by holder.

Our model ceramic system consists of three phases: YSZ, Alumina, and Spinel, and six types of grain boundaries (Fig. 1). HAADF-STEM images indicate presence of high Z elements at Spinel-Spinel and Alumina-Alumina grain boundaries (Figs. 2a-b), while a dark contrast at YSZ-YSZ boundary indicate either presence of lower Z elements or reduction in HAADF intensity due to structural distortion at grain boundaries caused by charge-compensating anion vacancies (Fig. 2c). As for the heterointerfaces, Spinal-Alumina grain boundary also show presence of high Z elements (Fig. 2d). On the other hand, YSZ-Spinel and YSZ-Alumina heterointerfaces do not show any elemental segregation indicating there is no driving force for segregation to occur; thus, these boundaries are thermodynamically stable (Figs. 2e-f). Using Energy Dispersive Spectroscopy (EDS), we previously confirmed the

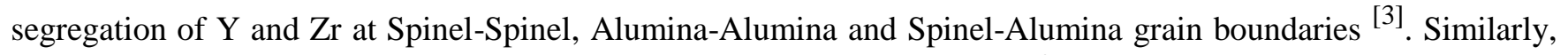
our EDS results confirmed the presence of $\mathrm{Al}$ at YSZ-YSZ grain boundary ${ }^{[3]}$.

We observe prominent clustering of Y/Zr segregants at the Spinel-Alumina heterointerface (Fig. 2d). This clustering of segregated atoms is towards the Alumina side of GB; while on the Spinel side, the segregants appear to be substituting the host cation sites (Fig. 2d). Alumina-Alumina grain boundary also shows some weak clustering effect, i.e. some segregants are clustered while others are more ordered (Fig. 2a). In contrast, the arrangement of segregated cations at the Spinel-Spinel grain boundary is relatively ordered, with Y/Zr cations substituting the host cation sites (Fig. 2b). At the YSZ-YSZ interface, we again observe some discontinuous arrangement of Al segregants (Fig. 2c). Many material properties, such as fracture, can be directly impacted by the spatial arrangement of the grain boundaries and chemical segregation. Thus, this study highlights not only the preferential elemental segregation at the grain boundaries of a multiphase ceramic processed by different techniques but also the variance in grain boundary structure, down to atomic resolution. We plan to supplement these experimental results with atomistic simulations in near future to gain more understanding of the grain boundary segregation phenomena. The elemental segregation and structural inhomogeneity observed at these grain boundaries would all contribute to macroscopic material properties, such as thermal conductivity and fracture toughness [4]. 



Figure 1. (a) There are 6 types of boundaries in a 3-phase ceramic with MgAl2O4, Al2O3, and 8YSZ. (b) BF-TEM image of SPS sample with randomly distributed grains of $\sim 500 \mathrm{~nm} . \mathrm{S}=\mathrm{MgAl} 2 \mathrm{O} 4, \mathrm{~A}=\mathrm{Al} 2 \mathrm{O} 3, \mathrm{Z}=8 \mathrm{YSZ}$.
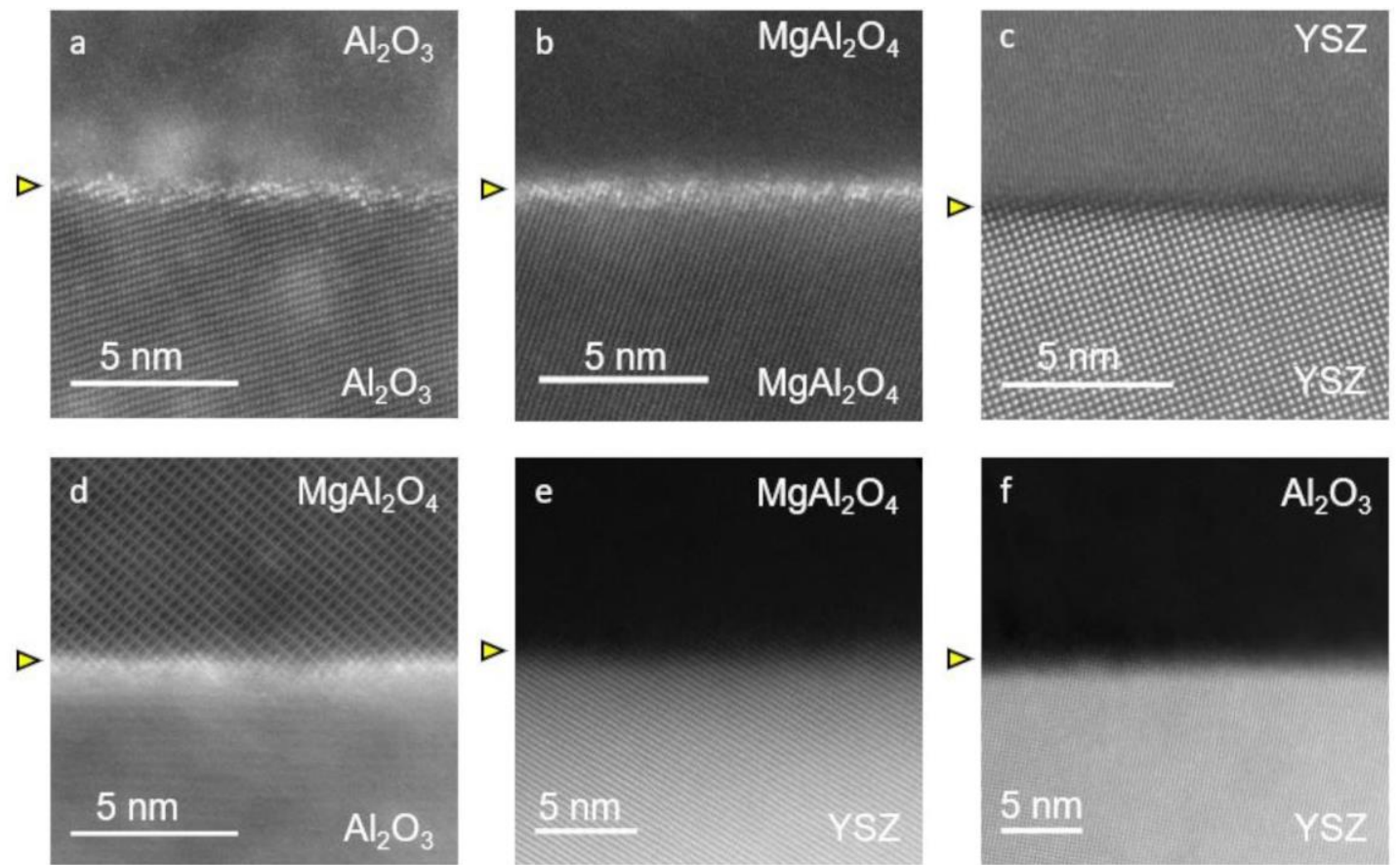

Figure 2. HAADF-STEM representative images of different types of grain boundaries in the 3-phase sintered ceramic. (a) Alumina - Alumina in SPS (b) Spinel - Spinel in conventional sintered (c) YSZ - YSZ in flash sintered (d) Spinel - Alumina in SPS (e) YSZ - Spinel in conventional sintered (f) YSZ - Alumina in SPS. The grain boundaries are indicated by arrows.

\section{References}

[1]. J.P. Kelly and O.A. Graeve, JOM 67 (2015), 29-33

[2]. M. Cologna, A.L.G Prette and R. Raj, J. Am. Ceram. Soc. 94 (2011), 316-319

[3]. K. Syed et al, Microsc. Microanal. 25 (2019), 2352-2353

[4]. This work is supported by NSF DMR Thermal Grant 442660-21130, and UCI faculty start up fund. The work was performed in the Irvine Materials Research Institute (IMRI). 